\title{
Efficacy and Adverse Effects of Self-Expandable Metal Stent Placement for Malignant Duodenal Obstruction: The Papilla of Vater as a Landmark
}

This article was published in the following Dove Press journal: Cancer Management and Research

\section{Chi-Huan Wu \\ Mu-Hsien Lee \\ Yung-Kuan Tsou \\ Wei Teng (D) \\ Cheng-Hui Lin \\ Kai-Feng Sung \\ Nai-Jen Liu (iD}

Department of Gastroenterology and Hepatology, Linkou Chang Gung Memorial Hospital, Taoyuan, Taiwan
Correspondence: Nai-Jen Liu

Department of Gastroenterology and

Hepatology, Linkou Chang Gung Memorial

Hospital, Taoyuan, Taiwan

Tel +886-3-328I200 Ext 8108

Fax +886-3-3272236

Email launaijn.tw@yahoo.com.tw
Purpose: Self-expandable metal stents are used for malignant duodenal obstruction. Outcomes between stents placed above and below the papilla of Vater differ, and no study has investigated these differences. We evaluated the efficacy and adverse events of stent placement in these two locations and reported our experience with self-expandable metal stent placement in patients.

Patients and Methods: We retrospectively analyzed the data of patients with unresectable metastatic cancers $(n=101)$, who underwent successful duodenal self-expandable metal stent placement between 2008 and 2018. Patients were divided into above and below the papilla of Vater groups. Patient demographics, technical and clinical outcomes, post-procedural morbidity, and stent patency were analyzed.

Results: Overall, 71 and 30 patients had intestinal obstruction above (including the papilla itself) and below the papilla of Vater and underwent successful stenting. Common bile duct obstruction was more common in the above-papilla group. Procedure time was similar between the groups, if an appropriate endoscope could facilitate stent placement in the below-papilla group. Both groups achieved symptomatic relief. Median stent patency duration was not significantly different between the groups; three patients had severe gastrointestinal bleeding due to postoperative vascular-enteric fistula.

Conclusion: Self-expandable metal stents can effectively relieve symptoms of duodenal obstructions located above and below the papilla of Vater. Duodenoscopes could facilitate stent placement if the obstruction is located below the papilla of Vater; if gastrointestinal bleeding occurs postoperatively, the possibility of vascular-enteric fistula formation should be considered. Keywords: enteric fistula, intestinal stricture, intestinal malignancy, bile duct obstruction, duodenoscope, gastrointestinal bleeding

\section{Introduction}

Duodenal obstruction is one of the most severe comorbidities caused by different malignancies, such as advanced local tumors in patients with pancreatic cancer or bile duct cancer or metastases that involve the peri-duodenal area. Typical symptoms include persistent nausea, vomiting, intolerance to oral intake, and weight loss and lead to malnutrition and delay in treatment of patients. Gastric bypass surgery with gastrojejunostomy has been proven effective for these patients. However, endoscopic placement of duodenal self-expandable metal stent (SEMS) has altered the management of malignant duodenal obstruction and improved quality of life of the patients. ${ }^{1-5}$ 
Anatomically, the duodenum is divided into four sections (superior, descending, horizontal, and ascending), with the common bile duct and the pancreatic duct joining at the major duodenal papilla. Given that these complex sections communicate with each other, the clinical background, technical challenge, and outcomes of treatment are different, depending on the level of duodenal obstruction in relation to the major papilla. For example, stent placement in patients with a malignant obstruction above the papilla of Vater could be performed easily with a gastroscope. However, stent placement in patients with malignant duodenal obstruction below the papilla of Vater can be difficult. No study has investigated these two types of duodenal obstructions separately. We describe our experience with patients with malignant duodenal obstructions above and below the papilla of Vater, who underwent SEMS placement. We also investigated the efficacy and adverse effects of the two stent placement procedures.

\section{Patients and Methods Study Design and Population}

This retrospective cohort study analyzed the data of consecutive patients who underwent enteric SEMS placement at our interventional endoscopy unit between 2008 and 2018. The study was approved by the Chang Gung Medical Foundation Institutional Review Board (IRB number: 201901718B0). Data acquisition and analysis were performed in accordance with the institution's guidelines and regulations. To comply with data privacy laws, all subjects were de-identified to preserve their privacy. Owing to the retrospective design of the study, the requirement for obtaining informed consent from the patients was waived by the ethics committee. The study was performed in accordance with the principles stated in the Declaration of Helsinki.

\section{Data Collection}

All data (including the endoscopy and radiology reports) were collected from patient medical records. Patients were divided into the following groups, according to the location of the stent placement: the above-papilla group (comprising patients with metallic stents placed covering the bulb to the papilla of Vater itself), and the below-papilla group (comprising patients with metallic stents placed below the papilla, including the lower part of the second section of the duodenum to the proximal jejunum). We collected data on patient demographics, tumor stage and origin, and biliary drainage before the procedure. Outcomes measured included technical and clinical success, symptom resolution, stent patency, re-interventions, and complications.

\section{Endoscopic Procedure}

We placed a large-bore (16G) nasogastric tube and left it on free drainage for at least 24 hours before the procedure, to ensure adequate gastrointestinal tract decompression. Uncovered metal stents, such as BonaStent (Standard Sci Tech, Seoul, Korea) and WallFlex Enteral Stent (Boston Scientific, Massachusetts, USA), were used for the procedures. Stent length ranged from $90 \mathrm{~mm}$ to $140 \mathrm{~mm}$. We anchored two metal stents for long intestinal strictures, when a single stent was inadequate. To overcome the difficulty of endoscope working length and the working channel diameter, we employed therapeutic gastroscopes (GIF-2T240, Olympus, Tokyo, Japan) for stenting in the above-papilla group, and colonoscopes (CF-H260AI, Olympus, Tokyo, Japan) or large-channel duodenoscopes (TJF-260V, Olympus, Tokyo, Japan) in the "below the papilla" group. Stent deployment was performed using the through-the-scope method and under fluoroscopic guidance. ${ }^{6}$ A water-soluble contrast medium was injected through a stone retrieval balloon catheter, which is used for stone retrieval during endoscopic retrograde cholangiopancreatography (ERCP). Initially, we employed a retrieval balloon catheter to cross the stricture utilizing a hydrophilic guidewire (0.035-inch Jag wire; Boston Scientific, Massachusetts, USA). After the balloon catheter successfully passed the stricture, we inflated the balloon and injected a contrast medium from the side hole of the balloon catheter, to measure the length of the obstructive lesion. The length of the SEMS to be placed was determined based on the estimated obstruction length, with at least 10-20 mm tumor-free intestinal margins on both sides. The catheter was then removed, and a SEMS was introduced over the guidewire across the stricture and deployed across it. A contrast medium was injected through the endoscope at the proximal end of the SEMS to confirm satisfactory stent placement.

\section{Follow-Up Information}

Technical success was defined as successful deployment of the SEMS across the obstruction. Clinical success was defined as symptomatic relief and measured as an improvement in the standardized gastric outlet obstruction scoring system (GOOSS) score (0: no oral intake, 1: 
liquids only, 2: soft solids, and 3: low-residue or full diet). ${ }^{7}$ Re-intervention was defined as any additional procedure performed for recurrent gastric outlet obstruction. Patients who had obstructive jaundice with the tumor compressing the common bile duct were identified in our study. Antitumor therapy after the procedure (systemic chemotherapy or regional radiotherapy) was also recorded. Moreover, the median stent patency rate and postoperative complications were also evaluated.

\section{Statistical Analysis}

Continuous variables were presented as the mean \pm standard deviations; categorical variables were presented as frequencies and percentages. The median was reported with a $95 \%$ confidence interval (CI). The GOOSS score before and at 1 week after stent placement was compared using the Wilcoxon signed-rank test. Differences in the data between the above-papilla and the below-papilla groups were compared using the Fisher's exact test for categorical variables, and the Mann-Whitney $U$-test for continuous variables. Stent patency rate was calculated by performing a Kaplan-Meier analysis. Statistical analyses were performed using SPSS Statistics, version 25 (IBM, Armonk, NY, USA). A two-sided p-value of $<0.05$ was considered statistically significant.

\section{Results}

\section{Baseline Information}

Overall, 103 patients were referred to our department for endoscopic stenting for symptomatic malignant duodenal obstruction and underwent palliative SEMS insertion. Of these, 71 patients had intestinal obstruction above the papilla of Vater (including the papilla of Vater itself), whereas 32 patients had intestinal obstruction below the papilla of Vater. Amongst the 32 patients with duodenal obstruction below the papilla of Vater, two patients experienced SEMS placement failure due to an acute angulation and an inability to pass the guidewire. Technical success rate was $100 \%$ and $93 \%$ in the above-papilla and the below-papilla groups, respectively. In the above-papilla group, 30 patients underwent stenting with the Bonastent stent (Standard Sci Tech, Seoul, Korea), whereas 41 patients underwent stenting with the WallFlex Enteral Stent (Boston Scientific, Massachusetts, USA). In the below-papilla group, 16 and 14 patients underwent stenting with the Bonastent and the WallFlex Enteral stents, respectively. Baseline characteristics of the patients with malignant duodenal obstruction who underwent successful SEMS placement are shown in Table 1. Age, sex, and tumor stage were similar between the two groups. Pancreatic cancer contributed towards half of the cases of duodenal obstruction in both the above-papilla and the below-papilla groups. The above-papilla group was more likely to have duodenal obstructions caused by bile duct and duodenal cancers. Conversely, the below-papilla group was more likely to have duodenal obstructions caused by metastatic cancers (including colon, lung, ovarian, cervical, and urinary bladder cancers), and were less likely to have duodenal obstructions caused by bile duct and duodenal cancers. It was interesting to note that the abovepapilla group was at an increased risk of having common bile duct obstruction. In the above-papilla group, 68 patients $(95.77 \%)$ had common bile duct obstruction before stent placement, with 44 patients undergoing common bile duct stent placement, and 24 patients undergoing percutaneous transhepatic cholangiography and drainage (PTCD) before duodenal stent placement. However, only 6 patients $(20.00 \%)$ in the below-papilla group had

Table I Characteristics of Patients with Malignant Duodenal Obstruction Who Underwent Successful Self-Expandable Metal Stent Placement

\begin{tabular}{|c|c|c|c|}
\hline & Above the Papilla $(N=7 I)$ & Below the Papilla $(\mathbf{N}=30)$ & P-value \\
\hline Sex, male:female, n:n & $36: 35$ & $17: 13$ & $P=0.673$ \\
\hline Age, years & $63 \pm 16^{*}$ & $62 \pm 12 *$ & $P=0.407$ \\
\hline TNM stage, III:IV, n:n & $26: 45$ & $7: 23$ & $P=0.248$ \\
\hline \multicolumn{4}{|l|}{ Underlying malignancy } \\
\hline Pancreatic cancer & $36(50.70 \%)$ & $16(53.33 \%)$ & $P=1.000$ \\
\hline Common bile duct and duodenal cancers & $31(43.66 \%)$ & $2(6.67 \%)$ & $P<0.001$ \\
\hline Metastatic cancers & $4(5.63 \%)$ & $12(40.00 \%)$ & $\mathrm{P}<0.00 \mathrm{I}$ \\
\hline Bile duct obstruction & $68(95.77 \%)$ & $6(20.00 \%)$ & $P<0.001$ \\
\hline
\end{tabular}

Note: *Values are shown in mean \pm standard deviation (percentage).

Abbreviation: TNM, tumor node metastasis. 
common bile duct obstruction, with 2 patients undergoing common bile duct stent placement, and 4 patients undergoing PTCD.

\section{Efficacy Evaluation}

Clinical success with improvement in oral intake was achieved in all patients with successful stent placement. The mean procedure time was slightly longer in the belowpapilla group than in the above-papilla group, but no significant differences were observed (23.12 minutes in the below-papilla group and 18.62 minutes in the above-papilla group). The mean GOOSS score improved from 0.32 (preprocedure) to 2.32 (post-procedure) in the above-papilla group and from 0.37 (pre-procedure) to 2.37 (postprocedure) in the below-papilla group, showing a statistically significant difference in both groups $(p<0.001)$.

The median stent patency duration for the above-papilla and the below-papilla groups was 123 days (95\% CI: 106-141 days) and 156 days (95\% CI: 113-198 days), respectively. The Kaplan-Meier curve is shown in Figure 1, and no significant differences were observed between the two groups. Recurrent obstructive symptoms occurred in 7 out of the 71 patients $(9.85 \%)$ in the above-papilla group, and 3 out of the 30 patients $(10 \%)$ in the below-papilla group. All recurrent obstruction cases were related to tumor in-growth. In the above-papilla group, all 7 patients underwent SEMS replacement. In the below-papilla group, 2 patients had to undergo palliative gastrojejunostomy, while the remaining patient underwent additional enteral metal stent insertion. All patients achieved symptomatic relief after the rescue procedure. The main study outcomes between the aboveand the below-papilla groups are shown in Table 2 .

\section{Adverse Events}

No stent migration or duodenal perforation was found in any of the patients. However, 3 patients experienced serious gastrointestinal bleeding, including 1 patient from the above-papilla group, and 2 patients from the below-papilla group. In the first case, the patient had pancreatic cancer with duodenal bulb involvement and experienced massive hematochezia and hypovolemic shock 3 days after the procedure. Upper gastrointestinal endoscopy revealed a large amount of blood in the duodenal bulb. Emergency angiography was performed and revealed contrast medium extravasation from the gastroduodenal artery into the duodenal bulb, just behind the SEMS (Figure 2).

The second case was a patient with pancreatic cancer with involvement of the third section of the duodenum. The tumor had encased the superior mesenteric artery. The patient had massive hematemesis 2 weeks after SEMS placement. He underwent emergency upper gastrointestinal endoscopy, which revealed no active bleeding above the papilla of Vater. Emergency radiography showed a superior mesenteric artery aneurysm with contrast medium extravasation into the duodenal stent. The patient underwent emergency coil embolization treatment for the superior mesenteric artery aneurysm (Figure 3).

In the third case, the patient had ovarian cancer with retroperitoneal metastasis. Massive hematemesis was noted 6 months after SEMS placement. Upper gastrointestinal endoscopy revealed a small healing ulcer in the

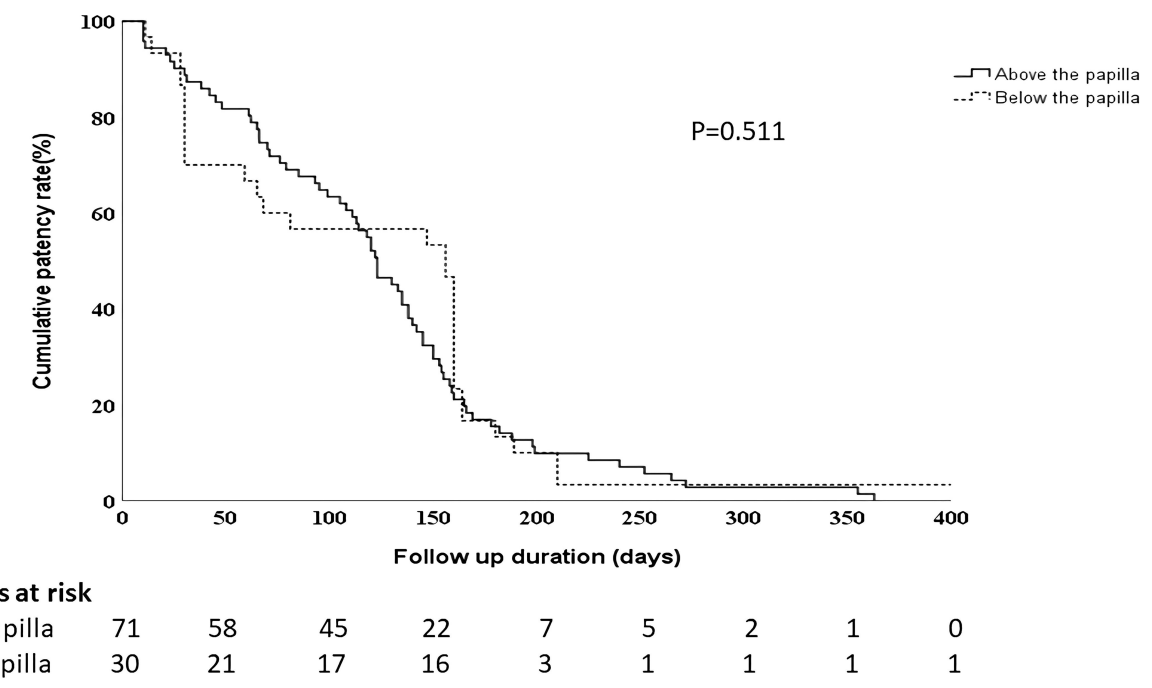

Figure I Kaplan-Meier curve showing the self-expandable metal stent patency rate of patients with malignant duodenal obstructions located above and below the papilla. 
Table 2 Outcomes of Patients with Malignant Duodenal Obstruction Who Underwent Successful Self-Expandable Metal Stent Placement

\begin{tabular}{|c|c|c|c|}
\hline & Above the Papilla $(\mathbf{N}=7 \mathrm{I})$ & Below the Papilla $(\mathbf{N}=\mathbf{3 0})$ & P-value \\
\hline Mean procedure time & $18.62 \pm 11.11 \mathrm{~min} *$ & $23.12 \pm 14.72 \mathrm{~min}^{*}$ & $P=0.123$ \\
\hline Mean stent length & $11.62 \pm 2.01 \mathrm{~cm}^{*}$ & $12.15 \pm 1.85 \mathrm{~cm}^{*}$ & $P=0.219$ \\
\hline Stent augmentation & $3(4.23 \%)$ & $2(6.67 \%)$ & $P=0.632$ \\
\hline \multicolumn{4}{|l|}{ GOOSS score } \\
\hline Mean score before stenting & $0.32 \pm 0.47^{*}$ & $0.37 \pm 0.49 *$ & \\
\hline Mean score after stenting & $2.32 \pm 0.75^{*}$ & $2.37 \pm 0.48^{*}$ & \\
\hline Chemotherapy after stenting & $25(35.21 \%)$ & $16(53.33 \%)$ & $P=0.121$ \\
\hline Anti-tumor radiation therapy & $8(26.67 \%)$ & $6(20.00 \%)$ & $P=0.344$ \\
\hline Number of complications & I (I.4I\%) & $2(6.67 \%)$ & $P=0.209$ \\
\hline Number of recurrences & 7 (9.86\%) & $3(10.00 \%)$ & $P=1.000$ \\
\hline Median stent patency duration $(95 \% \mathrm{Cl})$ & $\mid 23$ days $(|06-| 4 \mid$ days $)$ & I56 days ( 1 | $3-198$ days) & $P=0.778$ \\
\hline
\end{tabular}

Note: *Values are shown in mean \pm standard deviation (percentage).

Abbreviations: $\mathrm{Cl}$, confidence interval; GOOSS, gastric outlet obstruction scoring system.

antrum. Contrast-enhanced computed tomography (CT) imaging revealed active contrast medium leakage between the duodenum and the aorta, near the stent. The patient underwent aortic metal stent placement, which was performed by a cardiovascular surgeon (Figure 4).

\section{Discussion}

Patients with malignant duodenal obstruction may present not only with progressive symptoms, such as nausea and vomiting, but also with weight loss, early satiety, abdominal pain, cachexia, poor nutritional status, and severe dehydration. ${ }^{8}$ Apart from gastrojejunostomy, SEMS placement can be performed for the treatment of malignant duodenal obstruction.
Patients who underwent stent placement took a shorter time to achieve oral intake and had a shorter length of hospital stay than patients who underwent gastrojejunostomy. ${ }^{9-12}$ Endoscopic SEMS placement had lower morbidity and increased likelihood of patient recovery after the procedure. $^{13,14}$ In our hospital, almost all SEMS placement procedures were performed under conscious sedation with intravenous midazolam and fentanyl, to avoid the risks associated with general anesthesia for malnourished patients.

It was interesting to note that the cases of common bile duct obstructions were less frequent in patients with duodenal obstruction below the papilla, since a previous study evaluating SEMS placement in all sections of the duodenum
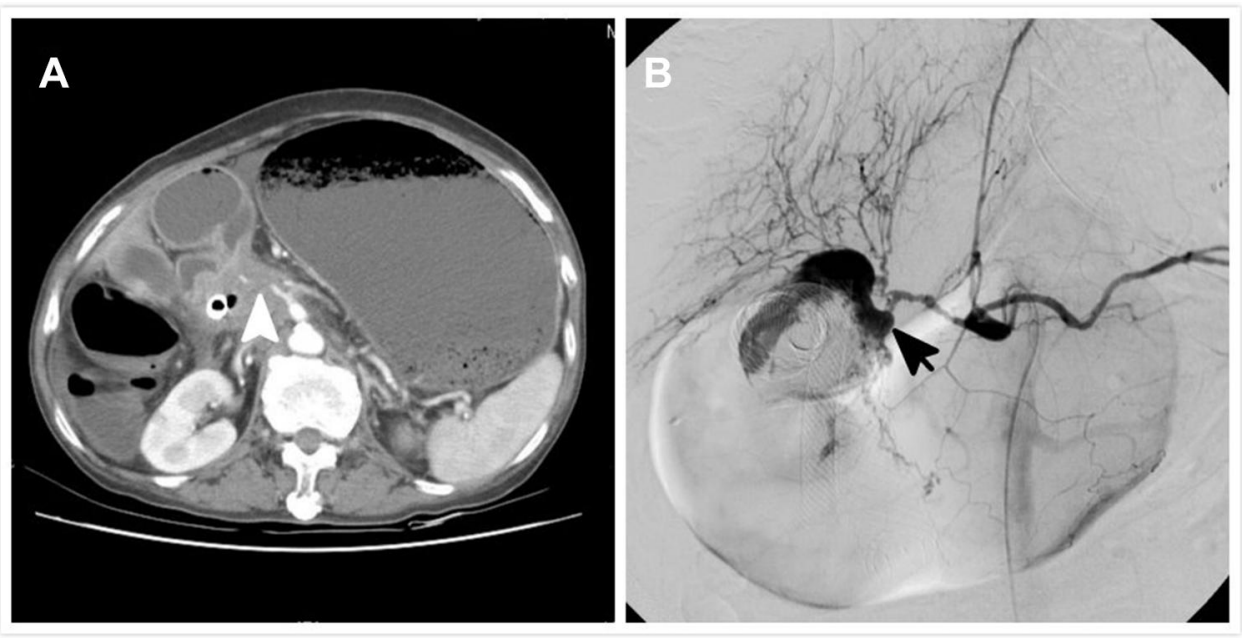

Figure 2 A 72-year-old patient with pancreatic cancer and duodenal obstruction. (A) Computed tomographic imaging before the procedure reveals a tumor encasing the duodenal bulb. Outline of the celiac and gastroduodenal arteries is irregular due to tumor invasion (white arrowhead). (B) Massive hematemesis is observed 3 days after stenting. Angiography reveals contrast medium extravasation into the duodenum near the stent (black arrow). 


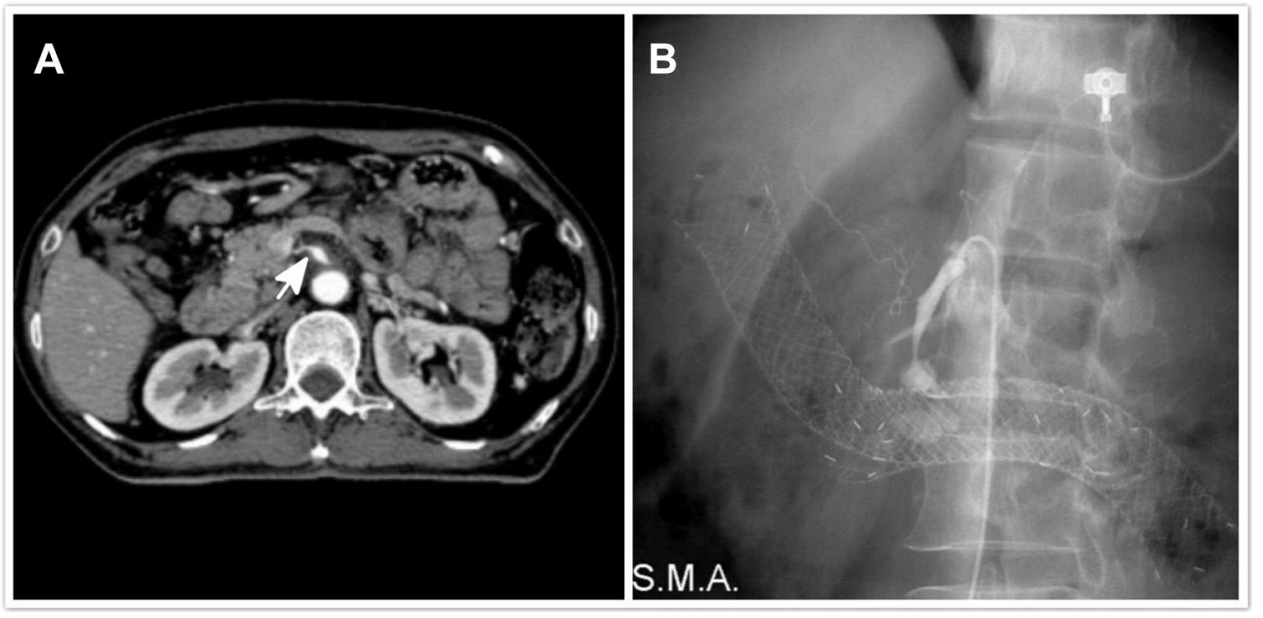

Figure 3 A 64-year-old patient with pancreatic cancer and duodenal obstruction. (A) Computed tomographic imaging before the procedure reveals a tumor encasing and invading the superior mesenteric artery. The vessel appears to have a fusiform shape (white arrow). (B) Hematemesis and hypovolemic shock are observed after stenting. Angiography reveals a superior mesenteric artery aneurysm near the stent.
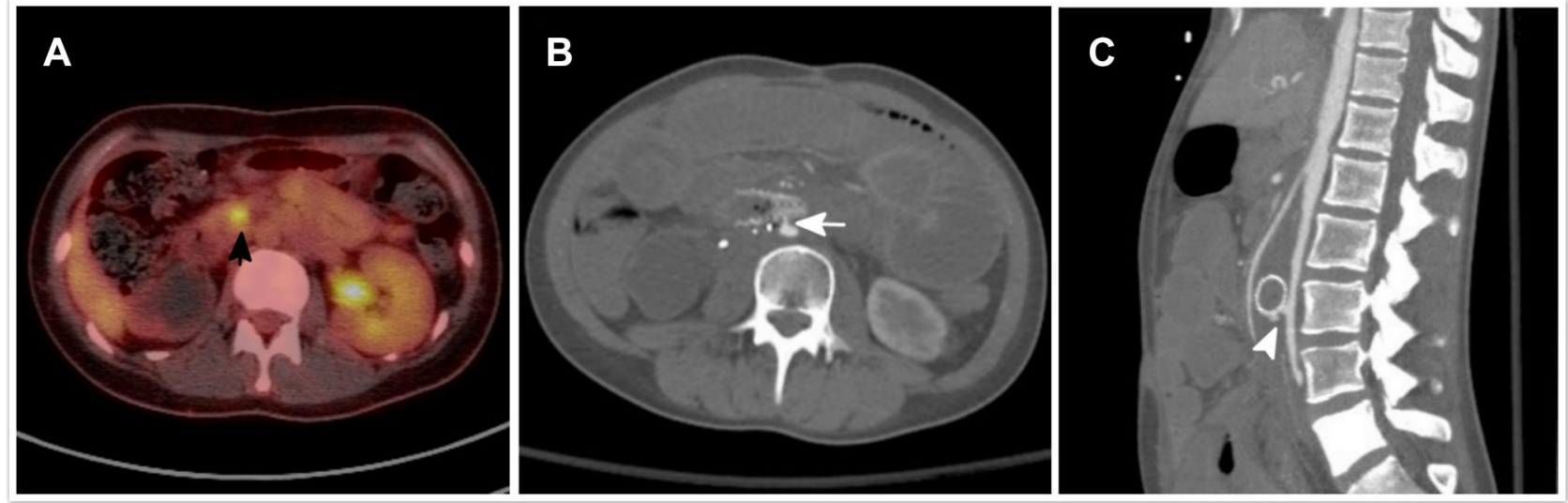

Figure 4 A 22-year-old patient with ovarian cancer and retroperitoneal involvement who underwent duodenal metal stent placement. (A) Positron emission tomographycomputed tomography performs before the procedure revealed a tumor infiltrating into the retroperitoneum, which causes an obstruction of the junction of the second and third portions of the duodenum (black arrow). The tumor is distant from aorta and the soft tissue around it is also tumor free. (B and $\mathbf{C}$ ) The patient develops a massive hematemesis 6 months after the stenting procedure. Contrast-enhanced computed tomography scan reveals an active contrast medium leakage between the duodenum and the aorta near the stent (white arrow and arrowhead).

had revealed that biliary obstruction occurs in $93 \%$ of patients. $^{15}$ Three types of duodenal strictures, according to their relation to the papilla of Vater, have been described. ${ }^{16}$ Type I stenosis occurs at the level of the duodenal bulb or the upper duodenal genu, but without involving the papilla. Type II stenosis affects the second section of the duodenum, with involvement of the papilla. Type III stenosis involves the third section of the duodenum, distal to and without the involvement of the papilla. Patients with type III obstruction (similar to the obstruction observed in the below papilla group in our study) only accounted for $6.25 \%$ of all cases in the study. ${ }^{16}$ Another study on duodenal SEMS placement and gastrojejunostomy has reported that none of the 5 patients who underwent gastrojejunostomy due to an obstruction of the third section of the duodenum had common bile duct obstruction. ${ }^{17}$ In our study, only $20 \%$ of the patients with malignant obstructions below the papilla required diversion of bile flow. However, bile duct diversion was required in $95 \%$ of the patients with duodenal obstructions above the papilla. This demonstrates that bile duct obstruction-related complications, especially biliary tract infections, may be less life threatening in patients with duodenal obstructions below the papilla.

Initially, we had hypothesized that duodenal SEMS placement below the papilla would be technically challenging due to the difficulty in approaching the stricture site and poor endoscopic handling due to the limited length of the endoscope. However, the difference in procedure time was not 
statistically significant between the two groups in our series. We performed colonoscopy for SEMS placement below the papilla as described previously in a study, ${ }^{18}$ in which colonoscopy was performed on 16 patients with distal duodenal obstruction (defined as the second half of the horizontal, ascending part of the duodenum, and the proximal jejunum), with a technical success rate of $93 \%$. In the last 3 years, we have tried using side-viewing duodenoscopes for SEMS placement in the below-papilla group (10 cases). Sideviewing duodenoscopes have been used in 31 patients with a technical success rate of $100 \% .{ }^{19}$ The availability of sideviewing duodenoscopes has also been reported in other studies. ${ }^{11,20}$ Conversely, SEMS placement in a shorter time has been demonstrated in a study that employed a forwardviewing rather than a side-viewing endoscope with a majority of the patients having an above-papilla duodenal obstruction (only one patient had duodenal obstruction in the third portion). ${ }^{21}$ Interestingly, procedure time with duodenoscopes was shorter than that with colonoscopes in the belowpapilla group $(28.35 \pm 15.30$ minutes using front-viewing colonoscope vs $12.70 \pm 4.45$ minutes using side-viewing duodenoscope, $\mathrm{P}<0.001)$, in our study. Side-viewing duodenoscopes provided multiple views, compared to frontviewing endoscopes (Figure 5). We were able to maintain adequate distance between the front end of the endoscope and the stricture site and then "cannulate" the stricture site by maneuvering the catheter and the guidewire, similarly to what is done during ERCP.

Severe gastrointestinal bleeding related to duodenal SEMS placement is rare, occurring in only $0.5 \%$ of the patients in a previous meta-analysis. ${ }^{1}$ Recently, a multicenter study has revealed that the rate of gastrointestinal bleeding is $0.4 \%{ }^{22}$ In our series, we encountered 3 patients with life-threatening bleeding due to a vascular-enteric fistula. Although the clinical
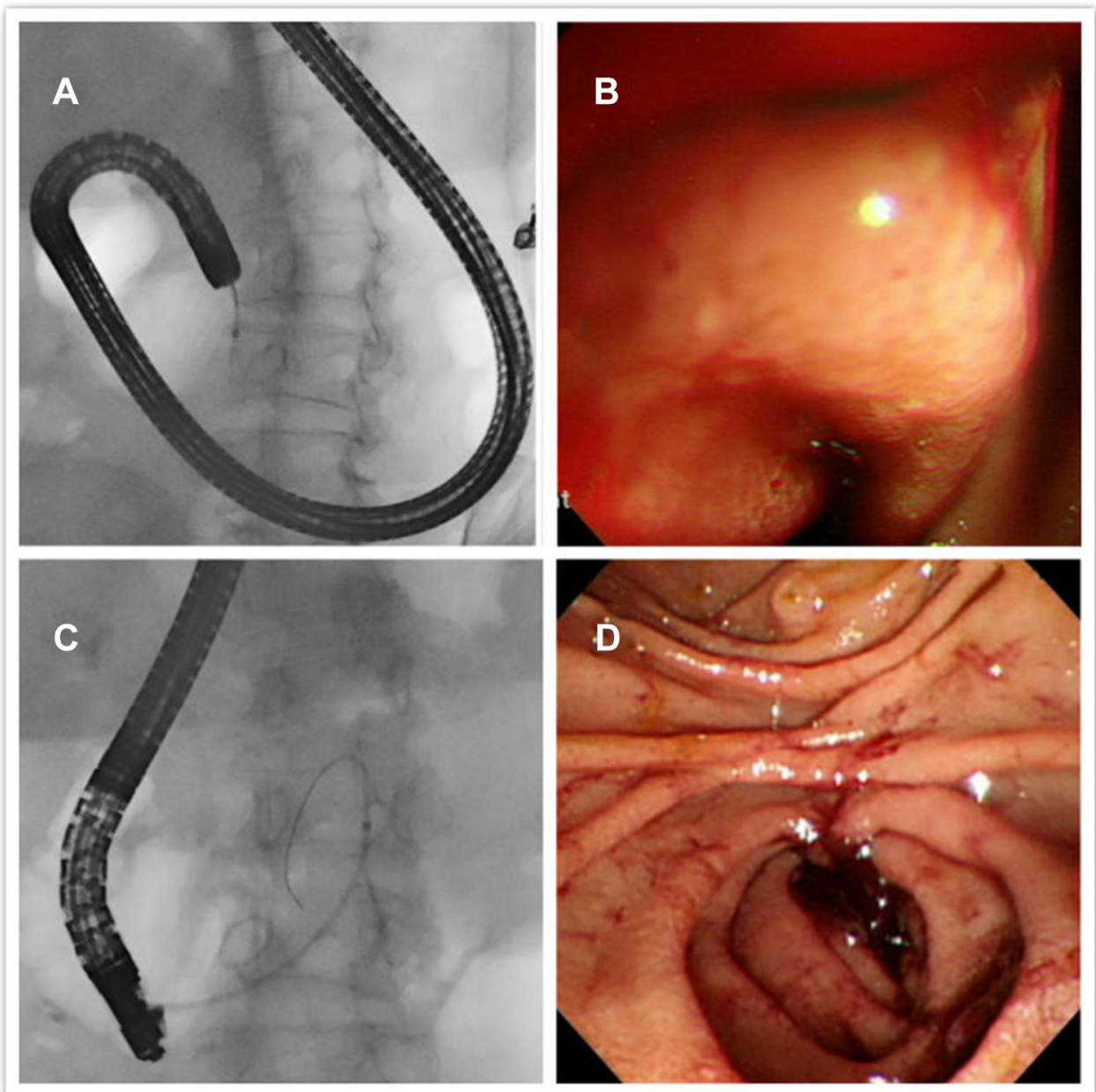

Figure 5 A case of malignant obstruction just below the papilla of Vater. (A and $\mathbf{B}$ ) The front-viewing endoscope is placed close to the stricture site. Endoscopic view is poor, even when the endoscope is twisted. (C and D) After shifting to a side-viewing duodenoscope, the duodenal stricture site can be seen clearly, and the guidewire can be inserted across the stricture site using an elevator. 
presentation was similar in the 3 patients, the time at which the complication occurred was different. We classified postSEMS placement bleeding into early $(<30$ days after the procedure) and late ( $>30$ days after the procedure). Two of our patients had early bleeding at 3 and 14 days, respectively, after the procedure. Pre-procedure CT revealed that the bleeding vessels were encased by the tumor, and their outline was irregular and blurred, suggesting that the vascular wall was invaded by the tumor. A case of massive bleeding within 72 hours of duodenal SEMS placement, similar to our case of early bleeding, has been reported. ${ }^{23}$ The author of the case report had hypothesized that the massive bleeding was caused due to a combination of tumor infiltration into the vessel, dilation of the stricture, and the presence of the selfexpandable metal duodenal stent. The same findings were also seen in the CT imaging performed before SEMS placement, in all our cases that presented with early bleeding postoperatively (Figures 2 and 3). The vessels were invaded by a tumor near the stricture site, which might be a risk factor for early bleeding. In the remaining case, complication occurred 6 months after the stent had expanded completely. Preprocedure positron emission tomography-computed tomography (PET-CT) imaging had revealed that the aorta had not been invaded by the tumor (Figure 4). Patients received radiation therapy for the local tumor 3 months after the SEMS placement. In a previous study on esophageal cancer, patients with esophageal obstruction could be treated with metal stents successfully; however, patients receiving radiation therapy were at a greater risk for developing an aorto-esophageal fistula due to radiation-induced necrosis and vascular changes in the esophageal wall. ${ }^{24}$ The fistula formation mechanism might be related to the following two factors: development of fibrosis in the submucosa, and thrombosis of the vasa vasorum due to the radiation therapy, resulting in large vessel perforation. $^{25-27}$ These factors might have contributed to the development of aorto-esophageal fistulas. The metal stent that we placed in the third section of the duodenum was similar to the one placed in the esophagus and close to the aorta. A case report demonstrated that metal stent placement in the third section of the duodenum led to aorto-duodenal fistula formation due to post-radiation duodenal strictures after a few years. ${ }^{28}$ Although radiation therapy is not routinely used for pancreatic or metastatic cancers in this anatomical region, it is considered for certain patients with malignant duodenal obstructions near large vessels, such as the aorta. Therefore, the risk of delayed bleeding due to the formation of vascularenteric fistulas should be considered before SEMS placement.
The limitation of our study was its retrospective and observational design. In our experience, surgical bypass is rarely selected as a treatment for our patients, due to poor clinical conditions. Choosing the type of anti-tumor therapy or best supportive care only also depends on the clinical situation of the patient. Our findings should also be verified in the clinical settings in the future.

\section{Conclusion}

SEMS placement below the papilla of Vater is a clinically effective option for treating malignant duodenal obstruction, similarly to SEMS placement above the papilla. Cases of common bile duct obstruction are less frequent in patients with duodenal obstruction below the papilla. Moreover, we found that sideviewing duodenoscopes could provide multiple views to facilitate SEMS placement below the papilla of Vater. If a major blood vessel near the stricture site is invaded by a tumor, or if the patient required radiation therapy for tumors near a large vessel, such as the aorta, metal stent placement should be considered keeping in mind the possibility of formation of a vascular-enteric fistula.

\section{Acknowledgments}

We would like to thank Editage (www.editage.com) for English language editing.

\section{Funding}

No funding was obtained for this study.

\section{Disclosure}

The authors declare that they have no competing interests for this work.

\section{References}

1. Dormann A, Meisner S, Verin N, Wenk Lang A. Self-expanding metal stents for gastroduodenal malignancies: systematic review of their clinical effectiveness. Endoscopy. 2004;36(6):543-550. doi:10.1055/ s-2004-814434

2. Graber I, Dumas R, Filoche B, et al. The efficacy and safety of duodenal stenting: a prospective multicenter study. Endoscopy. 2007;39(9):784-787. doi:10.1055/s-2007-966594

3. Piesman M, Kozarek RA, Brandabur JJ, et al. Improved oral intake after palliative duodenal stenting for malignant obstruction: a prospective multicenter clinical trial. Am J Gastroenterol. 2009;104 (10):2404-2411. doi:10.1038/ajg.2009.409

4. Endo S, Takiguchi S, Miyazaki Y, et al. Efficacy of endoscopic gastroduodenal stenting for gastric outlet obstruction due to unresectable advanced gastric cancer: a prospective multicenter study. $J$ Surg Oncol. 2014;109(3):208-212. doi:10.1002/jso.23486 
5. Sasaki R, Sakai Y, Tsuyuguchi T, et al. Endoscopic management of unresectable malignant gastroduodenal obstruction with a nitinol uncovered metal stent: a prospective Japanese multicenter study. World J Gastroenterol. 2016;22(14):3837-3844. doi:10.3748/wjg. v22.i14.3837

6. Baron TH, Harewood GC. Enteral self-expandable stents. Gastrointest Endosc. 2003;58(3):421-433. doi:10.1067/S00165107(03)00023-3

7. Adler DG, Baron TH. Endoscopic palliation of malignant gastric outlet obstruction using self-expanding metal stents: experience in 36 patients. Am J Gastroenterol. 2002;97(1):72-78. doi:10.1111/ j.1572-0241.2002.05423.x

8. Fukami N, Anderson MA, Khan K, et al.; ASGE Standards of Practice Committee. The role of endoscopy in gastroduodenal obstruction and gastroparesis. Gastrointest Endosc. 2011;74 (1):13-21. doi:10.1016/j.gie.2010.12.003.

9. Ly J, O’Grady G, Mittal A, Plank L, Windsor JA. A systematic review of methods to palliate malignant gastric outlet obstruction. Surg Endosc. 2010;24(2):290-297. doi:10.1007/s00464-009-0577-1

10. Jeurnink SM, van Eijck $\mathrm{CH}$, Steyerberg EW, Kuipers EJ, Siersema PD. Stent versus gastrojejunostomy for the palliation of gastric outlet obstruction: a systematic review. BMC Gastroenterol. 2007;7(1):18. doi:10.1186/1471-230X-7-18

11. Tringali A, Didden P, Repici A, et al. Endoscopic treatment of malignant gastric and duodenal strictures: a prospective, multicenter study. Gastrointest Endosc. 2014;79(1):66-75. doi:10.1016/j. gie.2013.06.032

12. Jeurnink SM, Steyerberg EW, van Hooft JE, et al. Surgical gastrojejunostomy or endoscopic stent placement for the palliation of malignant gastric outlet obstruction (SUSTENT study): a multicenter randomized trial. Gastrointest Endosc. 2010;71 (3):490-499. doi:10.1016/j.gie.2009.09.042

13. Fiori E, Lamazza A, Volpino $P$, et al. Palliative management of malignant antro-pyloric strictures. Gastroenterostomy vs. endoscopic stenting. A randomized prospective trial. Anticancer Res. 2004;24 (1):269-271.

14. Mehta S, Hindmarsh A, Cheong E, et al. Prospective randomized trial of laparoscopic gastrojejunostomy versus duodenal stenting for malignant gastric outflow obstruction. Surg Endosc. 2006;20 (2):239-242. doi:10.1007/s00464-005-0130-9

15. Vanbiervliet G, Demarquay JF, Dumas R, Caroli-Bosc FX, Piche T, Tran A. Endoscopic insertion of biliary stents in 18 patients with metallic duodenal stents who developed secondary malignant obstructive jaundice. Gastroenterol Clin Biol. 2004;28 (12):1209-1213. doi:10.1016/S0399-8320(04)95212-8

16. Mutignani M, Tringali A, Shah SG, et al. Combined endoscopic stent insertion in malignant biliary and duodenal obstruction. Endoscopy. 2007;39(5):440-447. doi:10.1055/s-2007-966327
17. Chang KB, Ye BW, Chou CK, et al. Outcomes of enteral metallic stent in patients with pancreatic carcinoma and gastric outlet obstruction: a single center experience. J Formos Med Assoc. 2020;119(1 Pt 2):238-246. doi:10.1016/j.jfma.2019.05.002

18. Jeurnink SM, Repici A, Luigiano C, Pagano N, Kuipers EJ, Siersema PD. Use of a colonoscope for distal duodenal stent placement in patients with malignant obstruction. Surg Endosc. 2009;23 (3):562-567. doi:10.1007/s00464-008-9880-5

19. Park JM, Min BH, Lee SH, et al. Feasibility of self-expandable metal stent placement with side-viewing endoscope for malignant distal duodenal obstruction. Dig Dis Sci. 2015;60(2):524-530. doi:10.1007/s10620-014-3343-8

20. Isayama H, Sasaki T, Nakai Y, et al. Management of malignant gastric outlet obstruction with a modified triple-layer covered metal stent. Gastrointest Endosc. 2012;75(4):757-763. doi:10.1016/j. gie.2011.11.035

21. Kumar V, Ghoshal UC, Mohindra S, Saraswat VA. Palliation of malignant gastroduodenal obstruction with self-expandable metal stent using side- and forward-viewing endoscope: feasibility and outcome. JGH Open. 2018;3(1):65-70. doi:10.1002/jgh3.12110

22. Hori Y, Naitoh I, Hayashi K, et al. Predictors of outcomes in patients undergoing covered and uncovered self-expandable metal stent placement for malignant gastric outlet obstruction: a multicenter study. Gastrointest Endosc. 2017;85(2):340-348.e341. doi:10.1016/j. gie.2016.07.048

23. Saleem A, Bakken J, Baron TH. Early massive bleeding after duodenal self-expandable metal stent placement for palliation of malignant gastric outlet obstruction (with video). Gastrointest Endosc. 2011;74(6):1426-1427. doi:10.1016/j.gie.2010.12.034

24. Bethge N, Sommer A, von Kleist D, Vakil N. A prospective trial of self-expanding metal stents in the palliation of malignant esophageal obstruction after failure of primary curative therapy. Gastrointest Endosc. 1996;44(3):283-286. doi:10.1016/S0016-5107(96)70165-7

25. Poon TP, Kanshepolsky J, Tchertkoff V. Rupture of the aorta due to radiation injury: report of a case and electron microscopic study. JAMA. 1968;205(12):875-878. doi:10.1001/ jama.1968.03140380079023

26. Alrenga D. Fatal hemorrhage complicating carcinoma of the esophagus. Report of four cases. Am J Gastroenterol. 1976;65(5):422-426.

27. Parikh MP, Sherid M, Panginikkod S, Rawal HA, Gopalakrishnan V. Radiation therapy-induced aortoesophageal fistula: a case report and review of literature. Gastroenterol Rep. 2016;4(2):165-167. doi:10.1093/gastro/gou081

28. Verhey P, Best A, Lakin P, Nachiondo J, Petersen B. Successful endovascular treatment of aortoenteric fistula secondary to eroding duodenal stent. J Vasc Interv Radiol. 2006;17(8):1345-1348. doi:10.1097/01.RVI.0000232499.04888.D0

\section{Publish your work in this journal}

Cancer Management and Research is an international, peer-reviewed open access journal focusing on cancer research and the optimal use of preventative and integrated treatment interventions to achieve improved outcomes, enhanced survival and quality of life for the cancer patient.
The manuscript management system is completely online and includes a very quick and fair peer-review system, which is all easy to use. Visit http://www.dovepress.com/testimonials.php to read real quotes from published authors. 